\title{
Ein Fall aus der anwaltlichen Praxis im Familien- und Erbrecht
}

\section{Zümrüt Turan-Schnieders}

Mitglied der Kommission Zivil-, Familien- und Erbrecht, Recht anderer Lebensgemeinschaften des djb, Rechtsanwältin, Hanau

Ich habe Ihnen einen Fall aus meiner Beratungspraxis mitgebracht, der geeignet ist aufzuzeigen, wie kulturelle Gewohnheiten und kulturelle Standards miteinander kollidieren können, welche Auswirkungen es haben kann, wenn zum Beispiel die familienrechtlichen Normen in ein etabliertes Herrschaftssystem eingreifen:

Meine Mandantin kommt aus der Türkei, aus dem modernen Teil der Türkei, aus Usak, sie hat die damals noch fünfjährige Pflichtschulausbildung dort absolviert. Ihre Ehe mit ihrem damals schon in Deutschland lebenden Ehemann wurde arrangiert. Auch nach sehr vielen und intensiven Gesprächen stellte sich nicht heraus, dass es eine Zwangsehe war. Mit 16 Jahren heiratete sie.

Ihr Ehemann lebte in Deutschland in einem Haus zusammen mit seinen Eltern und seinen Geschwistern. Meine Mandantin kümmerte sich - abgesehen vom Einkaufen - um den Haushalt. Sie war zuständig für das Kochen, Spülen, Putzen, alles, was in so einem Haushalt anfällt. Ihr wurde nicht ermöglicht, deutsch zu lernen, geschweige denn eine Ausbildung zu absolvieren. Die ältere Tochter, die selbst perfekt hessisch spricht, sagte im Anhörungstermin auf die Frage des Richters, warum ihre Mutter nicht deutsch sprechen würde: Sie hätte es nicht gebraucht, im Hause seien sie alle Türken.

Der ältere Bruder des Ehemannes und der Ehemann meiner Mandantin arbeiteten sehr fleißig, so dass sie genug Eigenkapital aufbringen konnten. So kauften die Brüder ein Mehrfamilienhaus für die Familie, in dem auch ein Ladengeschäft war. Dort zog eine türkische Bäckerei ein, meine Mandantin putzte die Bäckerei für 200,00 Euro pro Monat, das Geld wurde dem Ehemann übergeben. Auch das Kindergeld für die zwischenzeitlich drei Kinder wurde ausschließlich vom Ehemann verwaltet. Während der Ehe kauften die beiden Brüder ein weiteres Mehrfamilienhaus in der Türkei, das sie vollfinanziert haben. In das Grundbuch wurde der Vater des Ehemannes eingetragen, damit die Söhne für den Fall des Falles keine Nachteile erleiden.

Eines Tages stellte der Ehemann fest, dass er mit seiner Frau keine Gemeinsamkeiten mehr hätte - als ob sie jemals in diesem Familienverband hätten Gemeinsamkeiten entwickeln können. Er nimmt seine Frau zu seiner Anwältin mit, die junge Kollegin „berät“ die Eheleute. Weil es sich um eine einvernehmliche Ehescheidung handelte, nahm sie auch keinen Anstoß daran, dass der Ehemann, zwischenzeitlich Mandant der jungen Kollegin, seiner Ehefrau das Gesprochene übersetzt. Es herrschte noch relativer Frieden. Am Ende der Beratung waren sich die Eheleute „einig“, dass sie sich scheiden lassen und den Scheidungs- antrag in Deutschland einreichen wollten, weil das gesamte Scheidungsverfahren hier die Parteien nichts kostete. In der Türkei hätten sie für das Verfahren richtig zahlen müssen.

Die Rechtsanwältin des Ehemannes reichte den Scheidungsantrag auftragsgemäß beim zuständigen Gericht ein. Sie stellte den Scheidungsantrag und trug vor, dass für die Ehescheidung das türkische Recht gelte, weil beide Parteien noch die türkische Staatsangehörigkeit besitzen.

Auch das türkische Recht kennt die einverständliche Scheidung. Man muss zur Zerrüttung der Ehe etwas vortragen und dass die Ehe ein Jahr gedauert hat.

In der Antragsschrift wurde zu den Voraussetzungen der Zerrüttung nach dem türkischen Recht wie folgt vorgetragen:

„Die Parteien haben keinerlei Gemeinsamkeiten. Die Ehe wurde seinerzeit mehr oder weniger arrangiert und auch, wenn die Parteien über Jahre versucht haben, die eheliche Lebensgemeinschaft aufrecht zu erhalten, so sind sie nun an einen Punkt angekommen, an dem beide der Meinung sind, es ist besser, sich zu trennen. "

Gleichzeitig stellte sie einen Antrag auf Übertragung des Sorgerechts für die gemeinsamen Kinder auf den Kindesvater mit der folgenden Begründung:

„Da er als Alleinverdiener bei einem durchschnittlichen Nettoeinkommen von 2.200,00 Euro und entsprechender Schuldenbelastung durch den Bau des Mehrfamilienhauses einsam mit seinem Bruder nicht der Lage ist, auch noch ausreichend Unterhalt für seine Frau und die drei Kinder zu zahlen, sind die Parteien übereingekommen, dass die drei Kinder beim Vater, dem Antragssteller, bleiben sollen. Dem Antragssteller soll auch die elterliche Sorge übertragen werden, dies dient dem Kindeswohl und ist auch Wille der Kinder, sowie der Antragsgegnerin (...) Im Übrigen sind zumindest die beiden älteren Töchter alt genug, um selbstständig ihren Wunsch zu äußern, ob sie zur Mutter möchten oder nicht. Auch der jüngste Sohn, der im November 7 Jahre alt, hat sich entsprechend geäußert und möchte beim Vater, dem Antragssteller,

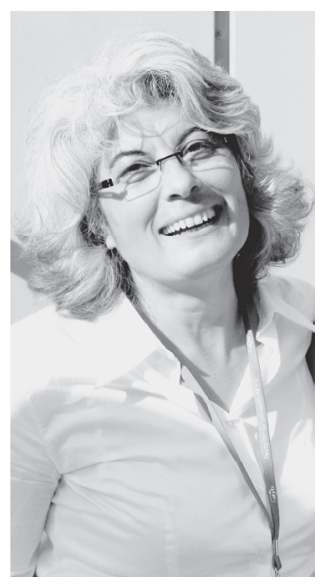

$\varangle$ Rechtsanwältin Zümrüt Turan-Schnieders (Foto: RM). 


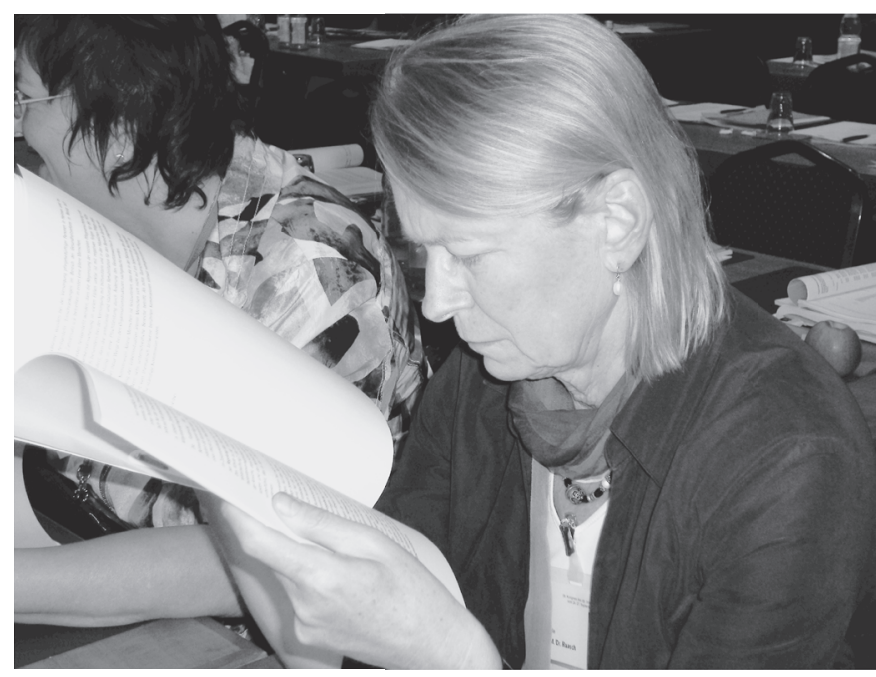

$\Delta$ Prof. Dr. Sibylle Raasch (Vors. der djb-Kommission Arbeits-, Gleichstellungs- und Wirtschaftsrecht 2005-2009) (Foto: AG).

bleiben, ohne selbstverständlich die Mutter, die Antragsgegnerin, zu verlieren".

Da beide Ehegatten türkische Staatsangehörige sind und damit auf ihre Ehe das türkische Recht Anwendung findet, dieses Recht aber keinen Versorgungsausgleich kennt, findet in solchen Fällen der Ausgleich der in der Ehe erworbenen Anwartschaften nur auf Antrag einer Partei statt. Davon ist in dem Scheidungsantrag keine Rede.

Meine Mandantin berichtete mir, dass sie diesen Zusammenhang nicht verstanden habe, aber davon ausgegangen sei, dass sie finanziell abgesichert sein würde, wenn sie geschieden werde. Weiter berichtete sie auf Nachfrage, dass das Konzept, das ihr Ehemann während der Beratung übersetzt hatte, vorgesehen habe, dass sie mit den Kindern aus der Ehewohnung ausziehe, aber der Vater das Sorgerecht erhalte, damit er mehr Geld habe.

Spätestens beim Diktat des Scheidungsantrages wurde es wohl der Kollegin bewusst, dass die Ehefrau zumindest eine sprachliche Hilfe im Gerichtsverfahren benötigen würde. Sie teilte dem Gericht mit, dass die Antragsgegnerin kaum Deutsch spreche und ihr ein Dolmetscher zur Seite gestellt werden müsse.

Haben wir als Anwältinnen, als Organ der Rechtspflege, nicht die Pflicht, zumindest die Beratung der einvernehmlichen Scheidung an der Stelle abzubrechen, wenn feststeht, dass die Ehefrau das Gesprochene nicht versteht? Geht unser Auftrag, die Interessen der Partei zu vertreten, so weit, dass wir eine offensichtliche - ich formuliere es sehr provokant - Entrechtung einer Frau erkennen, aber es geschehen lassen? Ich bin der Meinung, dass die Beratung eines Ehepaares auf keinen Fall ohne eine unabhängige Dolmetscherin stattfinden sollte, wenn einer der Ehepartner aus welchen Gründen auch immer nicht deutsch spricht.

Das für das Scheidungsverfahren zuständige Gericht stellte den Scheidungsantrag im Prozesskostenhilfeprüfungsverfahren zunächst nicht zu, weil ihm die Zustimmung der Antragsgegnerin zur erstrebten Sorgerechtsregelung fehlte. Gleichzeitig wurde angefragt, ob ein Antrag auf Versorgungsausgleich gestellt werden soll.
Diese Verfügung des Gerichts wurde an den Antragssteller weitergeleitet mit dem Zusatz, dass das Gericht die Zustimmung seiner Ehefrau zur angestrebten Sorgerechtsregelung verlangen würde. Seine Frau habe zwar der Unterzeichnerin gegenüber in der stattgefundenen Besprechung mitgeteilt, dass sie mit der angestrebten Regelung einverstanden sei, aber das Gericht würde eine schriftliche Zustimmung verlangen. Sie habe eine solche vorbereitet und würde ihrem Mandanten anheim stellen, diese von seiner Ehefrau unterzeichnen zu lassen und zurückzubringen. Das vorformulierte Schreiben lautete:

„Wie besprochen bestätige ich hiermit die Vereinbarung, dass das alleinige Sorgerecht unserer gemeinsamen Kinder, Kind 1, Kind 2, Kind 3, auf meinen Ehemann übertragen werden soll. Mein Mann wird keine Unterhaltsansprüche bei mir geltend machen. Das Umgangsrecht wird großzügig und nach freier Absprache untereinander gehandhabt. Mein Mann und ich sind uns insoweit einig. "

Diesen Brief legte der Ehemann meiner Mandantin vor und forderte sie auf, diese Bestätigung zu unterschreiben. Sie nahm das Schreiben an sich und weil sie es inhaltlich selbst nicht verstand, ging sie zu der Schwester ihres Ehemannes und bat sie, ihr das Schreiben zu übersetzen. Die Schwägerin, die ansonsten gut deutsch spricht, versuchte das Schreiben zu übersetzen, konnte aber mit dem Begriff „Sorgerecht“ nichts anfangen, so dass sie ihrer Schwägerin, meiner Mandantin, riet, sich an eine Dolmetscherin zu wenden.

Nun gibt es in dieser kleinen Stadt, in der die Familie lebt, keine Dolmetscherin. Die nächste Stadt liegt zwar nur sechs bis sieben Kilometer entfernt; meine Mandatin wusste aber nicht, mit welchem Bus sie in diese Stadt hätte fahren können. In ihrer Hilflosigkeit wandte sie sich an einen weiteren Bruder ihres Ehemannes und bat ihn, sie dorthin zu bringen, damit sie sich das Schreiben übersetzen lassen kann. So kam sie zu einer Dolmetscherin in Hanau, die das Schreiben übersetzte, ihr den Begriff „Sorgerecht“ erklärte und ihr dringend riet, sich selbst anwaltlich vertreten zu lassen. So geriet sie durch ihren Schwager zu mir in die Kanzlei.

In dem ersten Gespräch saßen neben meiner Mandantin auch der Bruder des Ehemannes und dessen Ehefrau. Die Fragen, die ich meiner Mandantin stellte, wurden zunächst konsequent vom Bruder des Ehemannes beantwortet. Er versuchte, auch mir das Konzept seines Bruders zu erklären. Ich bat ihn, sich aus dem Gespräch herauszuhalten und teilte ihm zunächst freundlich mit, dass ich seine Dolmetschertätigkeit nicht für nötig hielte, da ich auch der türkischen Sprache mächtig sei. Dennoch dominierte der Schwager weiterhin das Gespräch mit der Begründung, dass er ja wohl den Sachverhalt sehr gut kennen würde und er mir die Informationen besser geben könne.

Ich wollte ihn auf der einen Seite als Helfer meiner Mandantin nicht verlieren; gleichzeitig war mir aber sehr wichtig, die Version meiner Mandantin des Geschehens zu hören. Meine Mandantin machte den Eindruck, dass sie durchaus in der Lage wäre, auch alleine, ohne Hilfe des Schwagers zu mir zu kommen, wenn man ihr dies vorher erklären würde. So bat 
ich den Bruder, mit seiner Ehefrau den Besprechungsraum zu verlassen. Zunächst versuchte er über meine Mandantin doch noch in den Raum zu bleiben, in dem er zu ihr meinte, dass es wohl doch ihr Wunsch sei, dass er dabei sei. Hier tat meine Mandantin sich selbst den Gefallen und sagte ihm mit einer Entschlossenheit, die ich zu diesem Zeitpunkt von ihr nicht erwartet hätte, dass sie gerne mit mir alleine reden wollte.

Es stellte sich heraus, dass sie bis dahin überhaupt keine klare Vorstellung davon hatte, was diese Scheidung mit sich bringen würde. Vor allem wollte sie aber auf keinen Fall, dass der Sohn bei ihrem Mann verbliebe. Sie sagte auch gleichzeitig, dass sie auf ihre Kinder auf keinen Fall verzichten wolle. Am Ende des Gesprächs strebte auch sie eine Scheidung an mit der Begründung, dass, wenn er sie nicht mehr wolle, sie auch nicht mehr mit ihm verheiratet sein möchte. Aber das Sorgerecht sollte auf keinen Fall auf ihren Mann übertragen werden.

Auch die Frage des Versorgungsausgleiches erörterte ich mit ihr und riet ihr, einen entsprechenden Antrag zu stellen. Als es um die Regelung des Ehegattenunterhalts ging, sagte sie unmittelbar, ihr Mann hätte ihr gesagt, dass sie Sozialhilfe beziehen könne. Als ich sie fragte, ob es dann für sie in Ordnung sei und sie dadurch wohl möglich ihren gesicherten Aufenthaltsstatus in Deutschland verlieren könnte, stellte sich heraus, dass sie selbstverständlich über die Auswirkungen, nicht nur aufenthaltsrechtlicher Natur, keine Kenntnis hatte.

Da ein Sorgerechtsantrag gestellt war, fand ein Gespräch beim Jugendamt statt. In der Stellungnahme des Jugendamtes stand, dass die Eltern sich geeinigt hätten, zukünftig gemeinsam die elterliche Sorge für ihre Kinder ausüben zu wollen. Das Jugendamt teilte weiter mit, dass die Kinder die Hälfte der Woche beim Vater und die andere Hälfte bei der Mutter leben sollten und diese Regelung auch im Sinne der Eltern sei. Der Vater arbeite Schicht, daher solle es Absprachen zwischen der Mutter und dem Vater bezüglich der Zeiten, die die Kinder jeweils beim anderen Elternteil verbringen, geben. Eine Nachfrage bei der zuständigen Sachbearbeiterin des Jugendamtes ergab, dass auch hier keine unabhängige Dolmetscherin hinzugezogen worden war. Sie sagte, die Töchter hätten das Gespräch sehr gut gemanagt.

Aufgrund dieser Stellungnahme des Jugendamtes teilte das Gericht dem Antragsteller mit, dass er seinen Sorgerechtsantrag zurücknehmen möge.

Zwischenzeitlich forderte ich den Ehemann auf, Auskunft über seine Einkommensverhältnisse zu erteilen. Die Kollegin erteilte mir die Auskunft ohne Anerkennung einer Rechtspflicht. Nach der ersten Berechnung stellte sich heraus, dass der Ehemann sehr wohl in der Lage ist, an meine Mandantin und an die Kinder Unterhalt zu zahlen. Als mein Schreiben mit der Bezifferung auf dem Tisch lag, wollte der Vater doch noch das Sorgerecht für die Kinder alleine tragen, so dass der Sorgerechtsantrag nicht zurückgenommen wurde.

Inzwischen hatte sich meine Mandantin mit ihren Töchtern unterhalten, die sich dafür ausgesprochen haben, in dem Haus im Familienverbund weiter leben zu wollen, so dass nur der Antrag auf Übertragung des Sorge- bzw. Aufenthaltbestim-

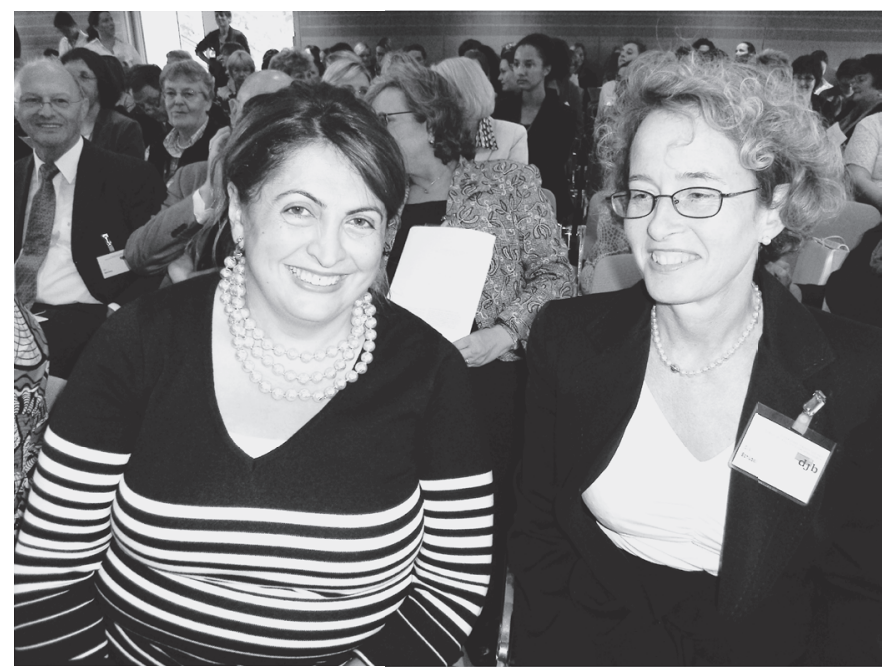

$\Delta$ Projektmanagerin Nimet Güller-Kaya (links) mit Bundesanwältin beim Bundesgerichtshof Eva Schübel (Foto: AG).

mungsrechts für den Sohn gestellt wurde. Gleichzeitig stellte ich für meine Mandantin einen Antrag auf Versorgungsausgleich.

Das Gericht hat das Aufenthaltbestimmungsrecht für die drei Kinder der Parteien dem Antragssteller alleine übertragen, die älteren Töchter betreffend mit Zustimmung meiner Mandantin, da sie sich dem Willen ihrer Töchter nicht widersetzen wollte. Die Begründung für die Übertragung des Aufenthaltbestimmungsrechtes bezüglich des kleinen Sohnes war folgende:

„Die Aufhebung der vollständigen gemeinsamen Sorge und die Übertragung des Aufenthaltbestimmungsrechtes entspricht dem Woble des Kindes am Besten. Die Anhörung aller drei Kinder ergab zunächst übereinstimmend, dass alle drei Kinder offensichtlich gerne zusammen leben wollen. Von daher ist es schon sehr bedenklich, wollte man die Kinder trennen. Weiterhin kommt hinzu, dass der Antragssteller eher in der Lage ist, zusammen mit seinen beiden Töchtern, die schon sehr reif sind, den Sohn zu fördern, weil dieser Teil der Familie ebenfalls die deutsche Sprache recht gut beherrscht. Das Kind ist gerade vor wenigen Tagen in die Schule gekommen und hat noch deutliche Sprachschwierigkeiten in der deutschen Sprache. Die Mutter ist, so bedauerlich es sein mag, nicht in der Lage, eine sprachliche Förderung zu leisten. Sicherlich ist sie in der Lage, das Kind zu versorgen, wobei dies aber auch dadurch außerordentlich gut möglich ist, wenn der Antragssteller auch oft das Kind zu der Mutter lässt. Dazu hat er seine Bereitschaft deutlich gemacht. Da es aber nun einmal leider sozusagen in letzter Sekunde zu einem Streit um das Aufenthaltbestimmungsrecht kam, war eine Entscheidung erforderlich geworden."

Der Fall endet zunächst hier. Das Urteil wurde mir erst vor ein paar Tagen zugestellt. Meine Mandantin lernt derzeit Deutsch und strebt eine Ausbildung an. Vielleicht begegne ich ihr in ein paar Jahren in einer völlig anderen Konstellation, als Deutsch sprechende und berufstätige Frau. Vor allem ihren Töchtern wünsche ich es. 